\title{
Compensation of the non-uniformity of back-lighting sources when digitising $X$-ray films for video-densitometric measures
}

\author{
Elisabetta M. Zanetti, Giordano Franceschini \\ Department of Industrial Engineering, University of Perugia, Perugia, Italy \\ Correspondence: Elisabetta M. Zanetti. Address: Department of Industrial Engineering, University of Perugia, Via \\ Duranti 1, 06125 Perugia, Italy. Email: elisabetta.zanetti@unipg.it.
}

Received: May 31, 2012

DOI : $10.5430 / j b g c . v 2 n 2 p 72$
Accepted: August 13, $2012 \quad$ Published: December 1, 2012

URL: http://dx.doi.org/10.5430/jbgc.v2n2p72

\section{Abstract}

Background: computerised image analysis and the building of electronic archives require the digitisation of x-ray films performed in the past, when direct digitisation was not yet available. Different techniques can be used, based on laser scanning or camera acquisition; both can be heavily impaired by the non-uniformity of the backlighting source. This work wants to develop a methodology, able to reduce this noise source.

Methods: The introduced algorithm is a step beyond the common compensation of backlighting non-uniformity through subtraction; in fact, each grey level is corrected taking into account not only its position on the back-lighting system and its raw value, but also the interaction between these two parameters.

Results: Given a certain acquisition system, the introduced algorithm has allowed to reduce the maximum grey level standard deviation, read on uniform grey level films, from 9.15 to 2.00 (on a scale from 0 to 255) against traditional subtraction techniques that achieved a minimum standard deviation equal to 5.39 .

Conclusions: The employed algorithm has proved to be effective in order to obtain same measured grey levels, almost independent from the position of the radiograph on the back-lighting system, and therefore makes $\mathrm{x}$ ray image digitisation more accurate.

\section{Key words}

Digital radiographs, Densitometry, X-ray images, Back-lighting systems

\section{I ntroduction}

In the last years, image processing techniques have quickly spread: being first confined to the informatic field, they have now become very useful means for the most various applications: chemistry, biology, engineering, geography, diagnostic medicine, etc. A typical application of image processing in the field of diagnostic medicine is related to the analysis of radiographic images ${ }^{[1]}$ : it is a long time since the consciousness arose that naked eye inspection of radiographic images is subjective and unable to appreciate the whole information contained in a radiograph. The first target has been the design of a device, able to give an objective measurement of grey levels: in 1901 the first step-wedge was used to determine bone-radiopacity ${ }^{[2]}$; different techniques are described by Stein ${ }^{[3]}$, Sanders (Dissertation, Pennsylvania State College, 
1937), and Bywaters ${ }^{[4]}$. The next step was the development of a system able to automatically measure a whole image as done by Mack and her co-workers ${ }^{[5]}$. In the following years, various instruments were enhanced and refined ${ }^{[6]}$ in order to improve measurement accuracy. An important evolution came in the seventies when radiographs have started to be acquired by means of a video-camera ${ }^{[7]}$, and successively with the advent of solid-state slide or image scanners, until nowadays when digitised radiographs are directly available. Nevertheless $\mathrm{x}$ ray film digitisation remains necessary when retrospective analysed are being performed ${ }^{[8]}$ or electronic archives are being built, or for implementing tele-radiology in places where conventional radiology is still used routinely as in rural healthcare centres of the developing world ${ }^{\text {[9] }}$. Different acquisition systems can be used: camera based ones, flatbed scanner, x-ray digitisers; up to now, none seems to have definitively overcome the other ones ${ }^{[9,10]}$, and all are affected by noise produced by the non-uniformity of the back-lighting source: Wenzel and Sewerin well outlined out the main sources of noise related to radiograph digitisation process ${ }^{[11]}$, while Audenino et al. demonstrated the importance of the non-uniformity of the back-lighting source to the end of results repeatability: it produces the dependency of measured grey levels on the position and orientation of the image on the back-lighting system ${ }^{[12]}$. This phenomenon has been seldom considered, but may not be insignificant: consider that using a common commercial back-lighting system with a uniform grey film overlaid on its top, the standard error of measured grey levels can reach 9.15 on a scale ranging from 0 to 255. This datum is not so known because usually background non-uniformity is measured without overlaying any film and, in this way, the standard error is underestimated (resulting to be very close to $0 \mathrm{OD}$ ) because of sensor saturation.

The technology has replied to the problem of background non-uniformity by using more and more sophisticated light sources, special reflection panels, and special transmission glasses. However the results are not so satisfactory; besides, the acquisition system comes to be very expensive; moreover, whenever too intense light sources are employed, problems of sensor saturation may arise.

An alternative to using more and more advanced hardware relies on the implementation of specific compensation algorithms through the creation of suitable software. The implementation of these algorithms determines, as a drawback, the reduction of the dynamic range that is the actual number of grey levels on disposal for image codification; this happens because the wider previous dynamic range was affected by more noise, and, as a consequence, could not be completely exploited. The advantage of the software approach is that greater accuracy can be obtained without requiring conspicuous investments.

Some attempts have been made in this sense in literature; most often the employed algorithm consists basically in the subtraction of the image of the back-lighting source from the image of interest (giving a certain offset in order to obtain all positive grey levels) ${ }^{[8,13]}$. Actually, as demonstrated in this work, this approach is quite rough: the problem is more complex than how it intuitively appears, and the simple subtraction algorithm does not bring a noticeable improvement. Another algorithm, introduced in ${ }^{[14]}$, seems to be able to effectively filter out randomly introduced noise. However it is opinion of the authors that a significant portion of digitisation noise is not randomly distributed, being related to the non-uniformity of the back-lighting source; a proof is given by the peculiar pattern of subtraction images obtainable when performing repeatability tests ${ }^{[11]}$; a further confirm was found by means of a rigorous statistical analysis ${ }^{[12]}$. More refined algorithms are summarized by Kim ${ }^{[15]}$; they take into account spatial frequency distribution ${ }^{[16]}$ or are based on segmentation ${ }^{[17,18]}$; maximum likelihood estimators are employed ${ }^{[15]}$ or a priori knowledge in relation to a given kind of images ${ }^{[18]}$. Such probabilistic or heuristic approaches are not required here because the source of noise is well known (the back-lighting system) and it can be quantified in a deterministic way (having assumed that the relative position between the camera and the back-lighting system remains fixed, in the case of camera acquisition).

For the aforementioned reasons a more complex algorithm has been here developed and introduced on the basis of a rigorous statistical analysis of the acquisition noise. Once set up, the algorithm has been tested in order to ascertain its effective capability of filtering noise out: the first test has been based on the measurement of the variance of grey levels of an image acquired after having overlaid a uniform OD film on the back-lighting system; the measurement is performed 
both before and after implementing the set up algorithm. A second test is based on the measurement of the variance of the average grey level of a certain area on a radiograph when many images are obtained through the acquisition of the same radiograph, differently positioned on the back-lighting system; again the measurement is performed both before and after implementing the set up algorithm.

\section{Materials and methods}

The experimental set up includes: a back-lighting system, a CCD camera (SNR $>62 \mathrm{~dB}$, resolution $2048 \times 2048$, sensitivity 0.01 lux at the sensor), equipped with a $60 \mathrm{~mm}$ lens, a frame grabber (2048×2048, $12 \mathrm{bit})$, and a monitor.

Images have been acquired in a dark room and the light came from the back-lighting system only.

Grey levels range was from 0 to 255, 0 corresponding to black, and 255 corresponding to white.

The software has been specifically created by means of Borland C++ compiler; it calculates an absolute grey level from each measured grey level, having taken into account the non-uniformity of the back-lighting system.

In order to set up the algorithm, 4 films have been used, sized A3, having a uniform optical density (OD), ranging from 0.3 to $1.2 \mathrm{OD}$ with a step equal to $0.3 \mathrm{OD}$. One or more films have been overlaid on the top of the back-lighting system in order to obtain images showing an almost uniform grey level equal to $0,0.3,0.6,0.9,1.2,1.5,1.8,2.1,2.4,2.7,3.0$ OD (e.g. the 1.5 OD image has been obtained overlaying both the 1.2 and 0.3 OD films on the top of the back-lighting system); each image has been acquired by means of the camera. As a result, a database is created and it is made of many images having an almost uniform grey level, whose value regularly decreases from the first image to the last one. In this case, the first two images (0 OD, 0.3 OD respectively) have been discarded because they gave rise to sensor saturation; the 0.6 OD image is the one showing the highest standard deviation of grey levels.

On the basis of all obtained data, a model can be created where the measured grey level of each point of an acquired image $\mathrm{G}_{\text {meas }}$ is associated to an absolute grey level $\mathrm{G}_{\text {abs }}$ that is the known optical density of the superimposed film. In this model, the dependent variable is $\mathrm{G}_{\text {meas }}$, while the significant independent variables have been identified by means of an analysis of variance, as explained in the following.

First of all a comparison has made between two models. In the first model, the input parameters are the $\mathrm{x}, \mathrm{y}$ co-ordinates and the optical density of the overlaid film:

$$
G_{\text {meas }}=f\left(x, y, O D_{\text {film }}\right)
$$

In the second model, the input parameters are the reference grey level $\left(\mathrm{G}_{\mathrm{ref}}\right)$ and the optical density of the superimposed film (OD); $\mathrm{G}_{\text {ref }}$ is the grey value measured in the same position as the measured grey level when the 0.6 OD film had been overlaid:

$$
G_{\text {meas }}=f\left(G_{\text {ref }}, O D_{\text {film }}\right)
$$

The second model would be more synthetic and therefore advantageous because many different couples (x, y) are associated with the same reference grey level; however, this model runs the risk of being less powerful if experimentally the same $G_{\text {ref }}$ values are not associated with the same $G_{\text {meas }}$ values.

The comparison of Table 1 and Table 2 confirms that the simplification of reducing two variables $(\mathrm{x}, \mathrm{y})$ to one single variable $\left(G_{r e f}\right)$ can be done without a significant deterioration of the model predictivity: in fact the estimated error is very 
similar in both cases (1.38 against 1.80 respectively) and the regression coefficient $\mathrm{R}^{2}$ is greater than 0.99 for both models. The physical meaning of this observation is that the variation of the grey level produced by film superposition is not dependent on the position of the examined point on the back-lighting system. This consideration, although intuitive, could not be rigorously assumed a priori because of the complexity of considered optical phenomena: vignetting effect is produced not only by the non-uniformity of the back-lighting system, but also by other effects such as camera acquisition through its lens, at a given diaphragm aperture, which is a space dependent effect; however, in the present case, it plays a minor role, as statistically demonstrated in the preceding paragraph. Note that the error variance in Table 1 is assimilated to the interaction $\mathrm{x} \cdot \mathrm{y} \cdot \mathrm{OD}$ because there are no replications; on the contrary, the error variance in Table 2 is calculated on the basis of replications since different $(\mathrm{x}, \mathrm{y})$ couples are related to the same $\mathrm{G}_{\text {ref. }}$ In both cases, the obtained error values are in good agreement with those calculated in previous works ${ }^{[11]}$. Another relevant observation is that OD and $\mathrm{G}_{\text {ref }}$ interaction cannot be disregarded. However this is exactly what is done by those authors who compensate the non-uniformity of the back-lighting system by means of the simple subtraction of the image of the back-lighting source.

Table 1. Analysis of variance considering $x, y$, OD as input variables

\begin{tabular}{lllllll}
\hline & DOF & SS & MS & $\mathbf{F}_{\text {exper }}$ & $\mathbf{F}_{\mathbf{0 . 0 1}, \mathbf{n}, \boldsymbol{\infty}}$ & p-value \\
\hline $\mathrm{x}$ & 42 & 727002 & 17310 & 12560 & 1.58 & $<1 \mathrm{E}-3$ \\
$\mathrm{y}$ & 63 & 105043 & 1667 & 1210 & 1.46 & $<1 \mathrm{E}-3$ \\
$\mathrm{OD}$ & 8 & 7567826 & 945978 & 686398 & 2.51 & $<1 \mathrm{E}-3$ \\
$\mathrm{x} \cdot \mathrm{y}$ & 2646 & 24451 & 9.24 & 6.71 & 1.07 & $<1 \mathrm{E}-3$ \\
$\mathrm{x} \cdot \mathrm{OD}$ & 336 & 66905 & 199 & 144 & 1.19 & $<1 \mathrm{E}-3$ \\
$\mathrm{y} \cdot \mathrm{OD}$ & 504 & 4824 & 9.57 & 6.94 & 1.15 & $<1 \mathrm{E}-3$ \\
Err & 23920 & 32966 & 1.38 & & & \\
Tot & 27519 & 8529018 & & & & \\
\hline
\end{tabular}

Note. DOF= Number of degrees of Freedom; $\mathrm{SS}=$ Sum of squared residuals; $\mathrm{MS}=$ Mean square; Fexper $=$ Experimentally evaluated F ratio; $\mathrm{F}_{\mathrm{p}, \mathrm{n} 1, \mathrm{n} 2}=\mathrm{F}$ test statistics; Err= Error; Tot= Total

Table 2. Analysis of variance considering $\mathrm{G}_{\mathrm{ref}}$, $\mathrm{OD}$ as input variables

\begin{tabular}{lllllll}
\hline & DOF & SS & MS & F & $\mathbf{F}_{\mathbf{0 . 0 1 , n , \infty}}$ & p-value \\
\hline OD & 8 & 7567826 & 945978 & 467207 & 2.51 & $<1 \mathrm{E}-3$ \\
Gref & 45 & 837985 & 18622 & 10347 & 1.56 & $<1 \mathrm{E}-3$ \\
OD·Gref & 360 & 74505 & 207 & 102 & 1.18 & $<1 \mathrm{E}-3$ \\
Err & 27106 & 48702 & 1.80 & & & \\
Tot & 27519 & 8529018 & & & & \\
\hline
\end{tabular}

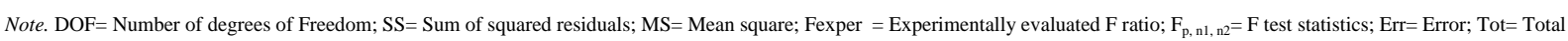

After having performed the analysis of variance, and accordingly with its results, an analytical model is built; it considers the measured grey level ( $\left.G_{\text {meas }}\right)$ as the output value, OD and the reference grey level $\left(G_{\text {ref }}\right)$ as input values. Actually, OD is strictly correlated with another value, characteristic of each film: the average measured grey level of the film itself $\left(\mathrm{G}_{\text {avg }}\right)$; for this reason $\mathrm{OD}$ has been substituted with $\mathrm{G}_{\text {avg }}$ in order to further simplify the analytical model: in this way the response curve of the optical sensor needs not to be kept into account, and all variables are homogenous for what concerns their measurement units. The following interpolation function is used in order to obtain the measured grey level $\left(\mathrm{G}_{\text {meas }}\right)$, from the Optical density $\left(\mathrm{G}_{\text {avg }}\right)$ and the reference grey level $\left(\mathrm{G}_{\mathrm{ref}}\right)$ :

$$
G_{\text {meas }}=0.23 G_{\text {avg }}+0.01 G_{\text {ref }} G_{\text {avg }}+0.18 G_{\text {ref }}-18
$$

The coefficients have been calculated through least squares fitting method. This interpolation function gives a correlation factor $r^{2}$ equal to 0.99 . Evidently it is a pure mathematical function, however, this polynomial interpolation has been 
considered to be fully adequate to describe the actual phenomenon since the obtained residual variance is comparable with the variance due to noise in the acquisition system. The coefficients appearing in the equation could vary in relation to different experimental results (that are $G_{\text {meas }}, G_{\text {avg }}, G_{\text {ref }}$ triples); they are expected to follow a normal distribution, as reported in ${ }^{[19]}$; also the estimated $G_{\text {meas }}$ value for given $G_{\text {avg }}$ and $G_{\text {ref }}$ is expected to follow a normal distribution, whose standard deviation grows moving from average input values to extreme input values ${ }^{[19]}$; as a rule of thumb, the highest variance of the estimated $\mathrm{G}_{\text {meas }}$ is lower that the error variance (MS of Err in Table 2) whenever data sample size is large enough (more than twenty data).

Actually the operator will use the inverse function since his aim is to produce an absolute grey level $\mathrm{G}_{\text {abs }}$ instead of a grey level biased by the non-uniformity of the back-lighting system; in other words he must be able to understand which film would produce $G_{\text {meas }}$ grey level, at the location where 0.6 OD film produced a grey level equal to $G_{\text {ref. }}$ This can be achieved by means of the following formula:

$$
G_{a b s}=G_{a v g}=\frac{G_{m e a s}-0.18 G_{r e f}+18}{0.23+0.01 G_{\text {ref }}}
$$

\section{Results}

The efficiency of the introduced method has been estimated recurring to the database of almost uniform OD images. First of all, the spatial standard deviation of measured grey levels has been calculated for each image belonging to this record. Thereafter, the measured grey levels of each image have been converted into absolute grey levels using eq (4), and the standard deviation of the absolute grey levels has been calculated for each image belonging to the record. As a result, the standard deviation of measured grey levels ranged from 4.13 to 9.15, while the standard deviation of absolute grey levels (related to the same images) ranged from 0.14 to 2.00. This has given proof of the efficiency of the introduced algorithm since, dealing with uniform OD films, the best measurement procedure is the one which gives the least standard deviation.

Another test has been made where absolute grey levels were obtained subtracting the image of the back-lighting system instead of using eq (1). Practically this model has been employed:

$$
G_{\text {abs }}=G_{\text {avg }}=G_{\text {meas }}-G_{\text {ref }}-\min _{\text {image }}\left(G_{\text {meas }}-G_{\text {ref }}\right)
$$

The last term is a constant value which has been added in order to avoid the obtainment of negative grey levels. This model has resulted in a spatial standard deviation of absolute grey levels ranging from 0 to 5.39, for the images belonging to the record. On the whole, it can be said that the introduced compensation algorithm, based on eq. (4), is more effective than traditional subtraction algorithms, based on eq. (5).

Further experiments have been made in order to practically assess the usefulness of the introduced algorithm, making use of ten radiographs of prosthetised human bones. Each radiograph has been overlaid on the back-lighting system in a random position for 6 times and each image has been acquired by means of the camera. One hundred measurement areas have been identified and the standard deviation over 6 measured grey levels has been calculated for each area (see Table 3 ); finally, the obtained standard deviations have been averaged over all areas. The experiment has been repeated after having converted measured grey levels into absolute grey levels by means of eq. (4), as shown in figure 1.The mean standard deviation of absolute grey levels has resulted to reduce up to -36\%. The mean standard deviation of absolute grey levels is therefore always smaller than the mean standard deviation of measured grey levels, and this is a second proof of the efficiency of the introduced algorithm. 
a)

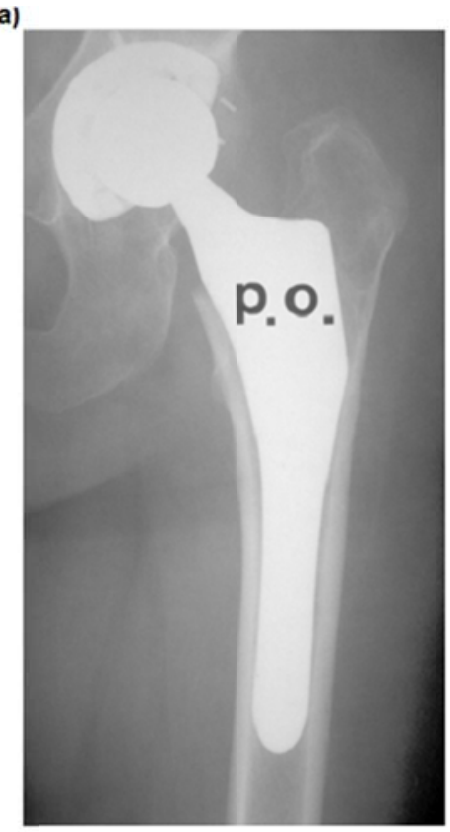

b)

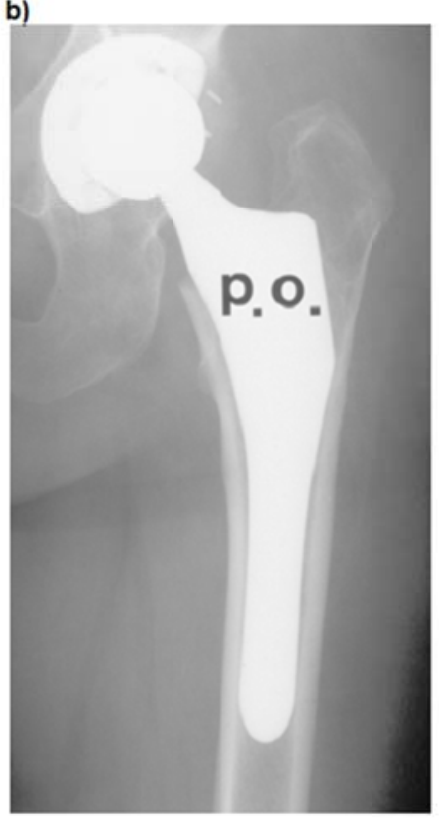

c)

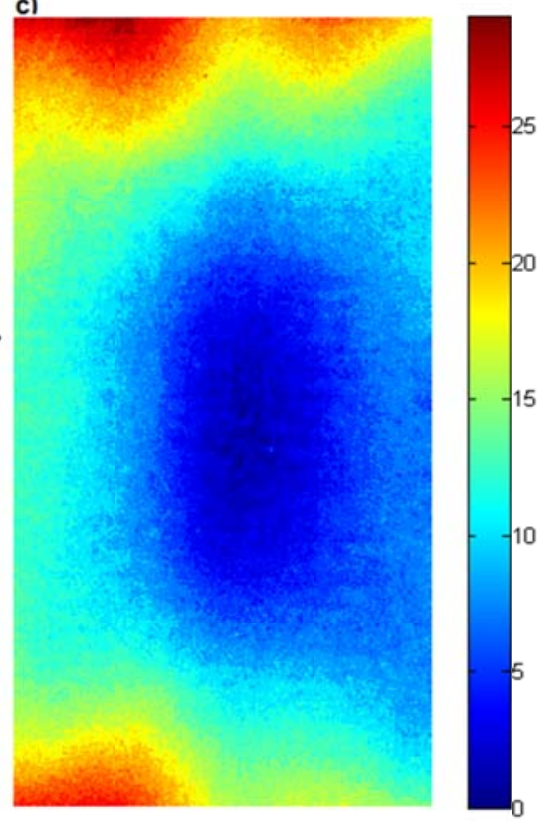

Figure 1. (a) Raw image; (b) compensated image; (c) map of grey level difference between the second image and the first one.

Table 3. Comparison of the standard deviations of measured grey levels, absolute grey levels (eq 4), and absolute grey levels obtained implementing background subtraction (eq 5)

\begin{tabular}{llll}
\hline Film density & St. Dev. $\mathbf{G}_{\text {meas }}$ & St. Dev. $\mathbf{G}_{\text {abs }}(\mathbf{e q ~ 4 )}$ & St. Dev. $\mathbf{G}_{\text {abs }}(\mathbf{e q} \mathbf{5})$ \\
\hline $\mathrm{OD}=3.0$ & 4.13 & 1.43 & 5.39 \\
$\mathrm{OD}=2.7$ & 4.32 & 1.50 & 5.22 \\
$\mathrm{OD}=2.4$ & 4.54 & 1.50 & 5.00 \\
$\mathrm{OD}=2.1$ & 4.77 & 1.45 & 4.76 \\
$\mathrm{OD}=1.8$ & 5.36 & 1.63 & 4.28 \\
$\mathrm{OD}=1.5$ & 6.12 & 1.72 & 3.58 \\
$\mathrm{OD}=1.2$ & 6.71 & 1.68 & 3.03 \\
$\mathrm{OD}=0.9$ & 7.63 & 2.00 & 2.50 \\
OD $=0,6$ & 9.15 & 0.14 & 0.00 \\
Average & 5.86 & 1.45 & 3.75 \\
Minimum & 4.13 & 0.14 & 0.00 \\
Maximum & 9.15 & 2.00 & 5.39 \\
\hline
\end{tabular}

Note. St. Dev.= Standard Deviation

\section{Discussion}

The aim of this work has been the setup of a new methodology for the compensation of back-lighting non-uniformity, since the simple background subtraction is not so effective ${ }^{[12]}$, as statistically demonstrated in this work: an analysis of variance has shown that, when a uniform OD film is overlaid on the top of the back-lighting system, measured grey levels are the result not only of the OD of the overlaid film and of the reference grey level but also of their interaction.

Commercial instrumentation has been employed, having chosen to reduce this source of noise through software, instead of recurring to sophisticated hardware. The introduced procedure does not require the use of expensive materials: just four uniform OD films are needed; the procedure should be followed by the operator the first time the acquisition system is set up and needs not to be repeated unless some changes in hardware have been made or a device has been moved; it is based 
on an interpolated function which must be considered as a characteristic of the acquisition system in use. The introduced methodology is a sort of filter that is advisable to apply after each acquisition in order to compensate for known background non-uniformity.

The methodology proved to be effective in reducing the variability of measured grey levels on a uniform film, however the measured standard deviation of absolute grey levels ( 0.14 to 2.00$)$ has resulted to be greater than the standard deviation due to acquisition noise (equal to 1.38 according to Table 1) ${ }^{[11]}$. A possible explanation may be that uniform OD films were not perfectly clean; besides, certain levels of optical density have been obtained overlaying more than one film, and a perfect adhesion was not easy to achieve.

The set up methodology has proven to be superior to simple background subtraction (eq. 5), even when $\mathrm{G}_{\text {ref }}$ has been identified with the grey level read on the 0.6 OD film (the film which resulted in the highest standard deviation of measured grey levels), and therefore the performance of subtraction algorithms is likely to have been overestimated: more often subtracted grey levels come from the acquisition of the back-lighting system with no film overlaid on, and the standard deviation of grey levels results to be underestimated due to sensors saturation.

The residual standard deviation of absolute grey levels obtained placing the X-ray image in different positions is still quite high (3.75): this result is not only due to the approximation introduced by the interpolation formula given by eq (4), but also to errors occurring in the identification of exactly the same reference area on radiographs differently positioned on the back-lighting system.

This methodology has been tested on one only hardware: also flat-bed scanners and film digitizers could be considered; however other authors demonstrated how the here-used acquisition set up performs similarly to the others, having the advantage of being less expensive ${ }^{[9,10]}$. The last aspect is significant, considering minor healthcare centres which could take benefit from teleradiology implementation ${ }^{[20]}$.

The set-up algorithm has been studied in order to eliminate one specific source of noise; further elaborations are needed in order to take into account the radiometric response and other vignetting effects; in the field of medical imaging; very promising algorithms are those introduced by Leemput et al. ${ }^{[18]}$ in relation to brain RM, based on 'a priori' knowledge, and the algorithm by Li et al. ${ }^{[17]}$ where a 'variational level set approach' to segmentation has been introduced.

\section{References}

[1] Kim TY, Son J, Kim KG. The recent progress in quantitative medical image analysis for computer aided diagnosis systems. Health Inform Res. 2011; 17(3): 143-9. PMid:22084808 http://dx.doi.org/10.4258/hir.2011.17.3.143

[2] Price WA. The science of dental radiography. Dental Cosmos. 1901; 43: 483-490.

[3] Stein I. Evaluation of bone density in roentgenogram by using ivory wedges. Am J Roentgenol \& Rad Therapy. 1937; 37: 678-682.

[4] Bywaters EGL. The measurement of bone opacity. Clinical Sciences. 1948; 6: 281-288. PMid:18904137

[5] Mack PB, Brown WN, Trapp HD. The quantitative evaluation of bone density. Am J Roentgenol. 1949; 61(6): 808-825.

[6] Mack PB, Vose GP, Nelson JD. New development in equipment for the roentgenographic measurement of bone density. Am J Roentgenol. 1959; 82(2): 303-310.

[7] Dickson RA, Paice F, Calnan JS. The quantitative colour television image analyser. J Bone Jt Surg 1973; 55B: $359-368$.

[8] Audenino AL, Calderale PM, Zanetti EM. Radiograph processing for quantitative assessment of bone remodelling. Med Eng Phys. 1996; 18(5): 382-389. http://dx.doi.org/10.1016/1350-4533(95)00059-3

[9] Salazar AJ, Camacho JC, Aguirre DA. Comparison Between Differently Priced Devices for Digital Capture of X-Ray Films Using Computed Tomography as a Gold Standard: A Multireader-Multicase Receiver Operating Characteristic Curve Study. Telemedicine and E-Health. 2011; 17(4): 275-282. PMid:21457011 http://dx.doi.org/10.1089/tmj.2010.0189

[10] Logeswaran, R; Choong, M K; Bister, M. Diagnostic quality of X-ray films digitised using conventional digital cameras. Imaging Science Journal. 2009; 57 (1): 1-7. http://dx.doi.org/10.1179/174313108X319388 
[11] Wenzel A, Sewerin I. Sources of noise in digital subtraction radiography. Oral Surg Oral Med Oral Pathol. $1991 ; 71:$ 503-8. http://dx.doi.org/10.1016/0030-4220(91)90441-E

[12] Audenino AL, Calderale PM, Zanetti EM. Application of the design of experiments for the evaluation of the robustness of video-densitometric measurements. Med Eng Phys 1997; 19 (5): 495-500. http://dx.doi.org/10.1016/S1350-4533(97)00016-7

[13] Brägger U. Digital imaging in periodontal radiography. J Clin Periodontol. 1988; 15: 551-557. PMid:3058754 http://dx.doi.org/10.1111/j.1600-051X.1988.tb02128.x

[14] Vos MH, Janssen PTM, Van Aken J, Heethaar RM. Quantitative measurement of periodontal bone changes by digital subtraction. J Period Res 1986; 21: 583-591. PMid:2947992 http://dx.doi.org/10.1111/j.1600-0765.1986.tb01496.x

[15] Kim SJ. Robust radiometric calibration and vignetting correction. IEEE Transactions on Pattern Analysis and Machine Intelligence. 2008; 30: 562-576. PMid:18276964 http://dx.doi.org/10.1109/TPAMI.2007.70732

[16] Zheng Y, Lin S, Kambhamettu C, Yu J, Kang SB. Single-Image Vignetting Correction. IEEE Transactions on Pattern Analysis and Machine Intelligence 2009; 31: 2243-2256. PMid:19834144 http://dx.doi.org/10.1109/TPAMI.2008.263

[17] Li C, Huang R, Ding Z, Gatenby C, Metaxas DN, Gore JC. A Variational Level Set Approach to Segmentation and Bias Correction of Images with Intensity Inhomogeneity. Med Image Comput Comput Assist Interv. 2008; 11: 1083-91. PMid:18982712

[18] Leemput KV, Maes F, Vandermeulen D, Suetens P. Automated Model-based bias field correction of MR images of the brain. IEEE Transactions on Medical Imaging 1999; 18: 885-896. PMid:10628948 http://dx.doi.org/10.1109/42.811268

[19] Milton JS, Arnold JC. Introduction to Probability and Statistics. London: McGraw Hill Higher Education, 2002.

[20] Moffatt JJ, Eley DS. The reported benefits of telehealth for rural Australians. Aust Health Rev. 2010; 34 (3): $276-81$. PMid:20797357 http://dx.doi.org/10.1071/AH09794 